\title{
Optical sensor interrogation with a blazed fiber Bragg grating and a charge-coupled device linear array
}

\author{
Alexander G. Simpson, Kaiming Zhou, Lin Zhang, Lorna Everall, and lan Bennion
}

\begin{abstract}
We present what is to our knowledge the first comprehensive investigation of the use of blazed fiber Bragg gratings (BFBGs) to interrogate wavelength division multiplexed (WDM) in-fiber optical sensor arrays. We show that the light outcoupled from the core of these BFBGs is radiated with sufficient optical power that it may be detected with a low-cost charge-coupled device (CCD) array. We present thorough system performance analysis that shows sufficient spectral-spatial resolution to decode sensors with a WDM separation of $75 \mathrm{\rho m}$, signal-to-noise ratio greater than $45-\mathrm{dB}$ bandwidth of $70 \mathrm{~nm}$, and drift of only 0.1 $\rho \mathrm{m}$. We show the system to be polarization-state insensitive, making the BFBG-CCD spectral analysis technique a practical, extremely low-cost, alternative to traditional tunable filter approaches. (C) 2004 Optical Society of America

OCIS codes: $\quad 050.1950,050.2770,060.2310,060.2340,060.2370$.
\end{abstract}

\section{Introduction}

Fiber-optic sensor techniques are of enormous interest because their small size, low cost, and ease of multiplexing make them ideal for embedding in composite materials. Traditionally, the problem with wavelength multiplexed optical sensors has been the recovery of information; bulky laboratory equipment cannot be used in the field since it is often prohibitively expensive and insufficiently rugged. The most promising reports to date involve variants of tunable filters to perform the optical interrogation. Common types of tunable filter include, the FabryPerot filter (an interferometric technique based on a tunable cavity between two mirrors), the sensorreceiver grating pair (the receiver grating is strained until the two gratings reflect the same wavelength, at which condition the measurand may be calculated), and the edge filter (the reflected signal from a sensor is passed through a linear intensity filter).

From the time that blazed fiber Bragg gratings (BFBG) were first reported, in 1990 by Meltz et al.,1 they have been demonstrated for applications, including wavelength division multiplexed (WDM) channel monitoring, ${ }^{2}$ erbium-doped fiber amplifier

The authors are with the Photonics Research Group, Aston University, Aston Triangle, Birmingham B4 7ET, United Kingdom. A. Simpson's e-mail address is appopt@george-simpson.co.uk.

Received 8 July 2003; accepted 3 October 2003.

0003-6935/04/010033-08\$15.00/0

(C) 2004 Optical Society of America
(EDFA) gain flattening, ${ }^{3}$ polarization discrimination, ${ }^{4}$ and edge filter manufacture. ${ }^{5}$ Several reports (for example, the Meltz et al. ${ }^{1}$ study) that show that BFBGs may be used to outcouple light from the core of the fiber have been published. This technique may be used as the basis for creating a diffractive fiber-optic spectrometer. ${ }^{6}$ Significant theoretical study on the phenomenon has been carried out, largely by Erdogan and associates. ${ }^{7-12}$

We present the first, to our knowledge, comprehensive study into the application of BFBGs as WDM sensor interrogators. We show that a BFBG may be used to outcouple light from the core of the fiber and may be detected with sufficient spectral-spatial resolution for it to be used in conjunction with a linear CCD array to decode a WDM sensor array. We investigate the bandwidth and resolution of the technique, its signal-to-noise ratio (SNR), drift, and WDM channel separation limits. We also show that the system is insensitive to polarization state, making the BFBG-CCD sensor interrogation method a highly practical, extremely low-cost, alternative to traditional sensor interrogation techniques.

Blazed Fiber Bragg Grating Theory: The index distribution in a BFBG structure is given by

$$
\Delta n(z, x)=\delta n \cos \left(\frac{2 \pi}{\Lambda} \cos \xi z-\frac{2 \pi}{\Lambda} \sin \xi x\right),
$$

where $\delta n$ is the amplitude of the index change, $\Lambda$ is the normal period of the grating, $z$ and $x$ are the coordinate axes defining the fiber orientation, and $\xi$ is 


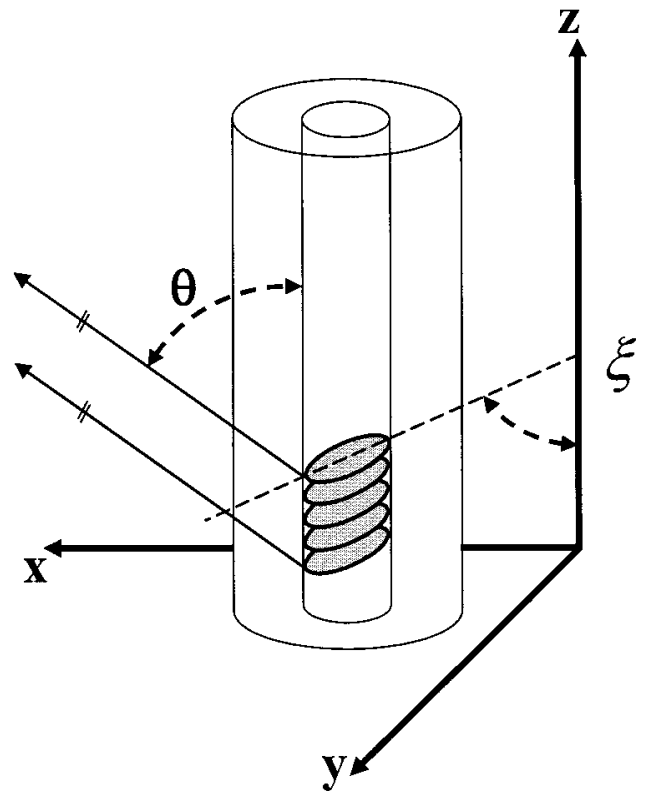

Fig. 1. BFBG geometrics showing the blaze angle and the radiation angle. The coordinate system used in this research.

the internal fringe tilt angle, as depicted in Fig. 1. The tilted structure permits light coupling between the core mode and the radiation modes, as shown by Fig. 2(a) in which a visible $633-\mathrm{nm} \mathrm{He}-\mathrm{Ne}$ laser is shown being outcoupled from a BFBG, and one introduces this structure during fabrication by either rotating the mask in a phase-mask exposure system or by rotating the fibre in a holographic inscription arrangement.

We have previously shown that the fringe angle of BFBGs in single-mode or multimode fiber, written by either fabrication technique, directly effects both the strength and the spectrum of the outcoupled radiation for a given grating period. ${ }^{13}$ Figure 2(b) illustrates this phenomenon for gratings written in single-mode fiber by the phase-mask technique. The radiation mode clearly increases in intensity with blaze angle; additionally, the Bragg resonance shifts toward longer wavelengths while its strength decreases. The radiation mode outcoupling also evolves as the blaze angle increases.

We have shown that the radiation mode vector is highly directional and that its exit angle is related to the fringe angle as follows ${ }^{14,15}$ :

$$
\cos \theta=\frac{\cos (\xi) \frac{\lambda}{\Lambda}-n_{\mathrm{eff}}(\lambda)}{n_{\mathrm{cl}}(\lambda)},
$$

where $\theta$ is the departure angle; $n_{\text {eff }}$ and $n_{\mathrm{cl}}$ are the refractive indices of the core and cladding, respectively; and $\lambda$ is the wavelength of the radiation. Owing to the cylindrical geometry of the fiber, the



(a)

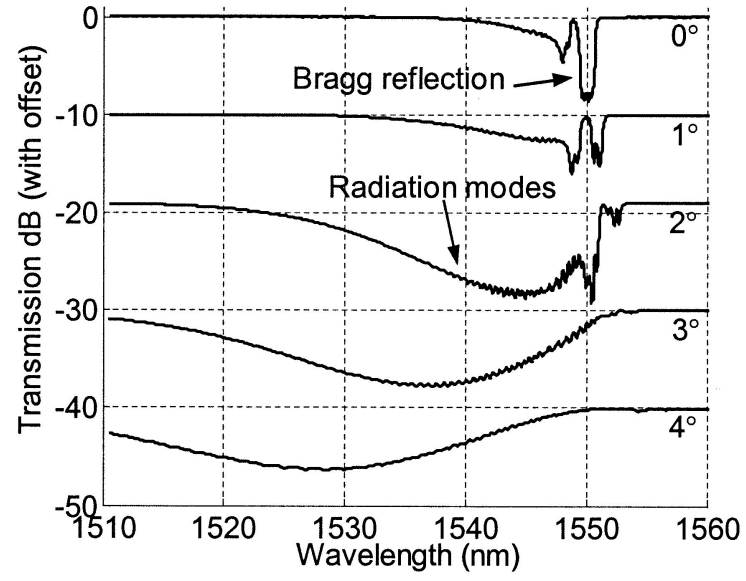

(b)

Fig. 2. (a) Photograph showing 633-nm He-Ne light being outcoupled from a BFBG. (b) Transmission spectra for BFBGs of increasing blaze angle.

internal fringe angle $(\xi)$ is related to external phasemask angle $\left(\xi_{\mathrm{PM}}\right)$ by ${ }^{16}$

$$
\xi=\frac{\pi}{2}-\tan ^{-1}\left(\frac{1}{n \tan \xi_{\mathrm{PM}}}\right) .
$$

Radiation modes from a uniform period BFBG are parallel in the $x-z$ plane but are divergent in the $y-z$ plane. Wagner et al. ${ }^{6}$ showed that for a chirped grating the radiation modes become slightly convergent with chirp rate, and in this study the calculated focal length, $f$, of a chirped BFBG was

$$
f \approx \frac{\Lambda^{2} \sin \left(\xi_{\mathrm{PM}}\right) \tan (\xi)}{\lambda \cos (\xi) C},
$$

where $C$ is the chirp rate in nanometers per centimeter.

Side detection of radiation modes offers many application-specific advantages ascribable to its highly predictable spectral-spatial relation; for example, in conjunction with a CCD detector array, the function of side-tapping light with high spatial reso- 


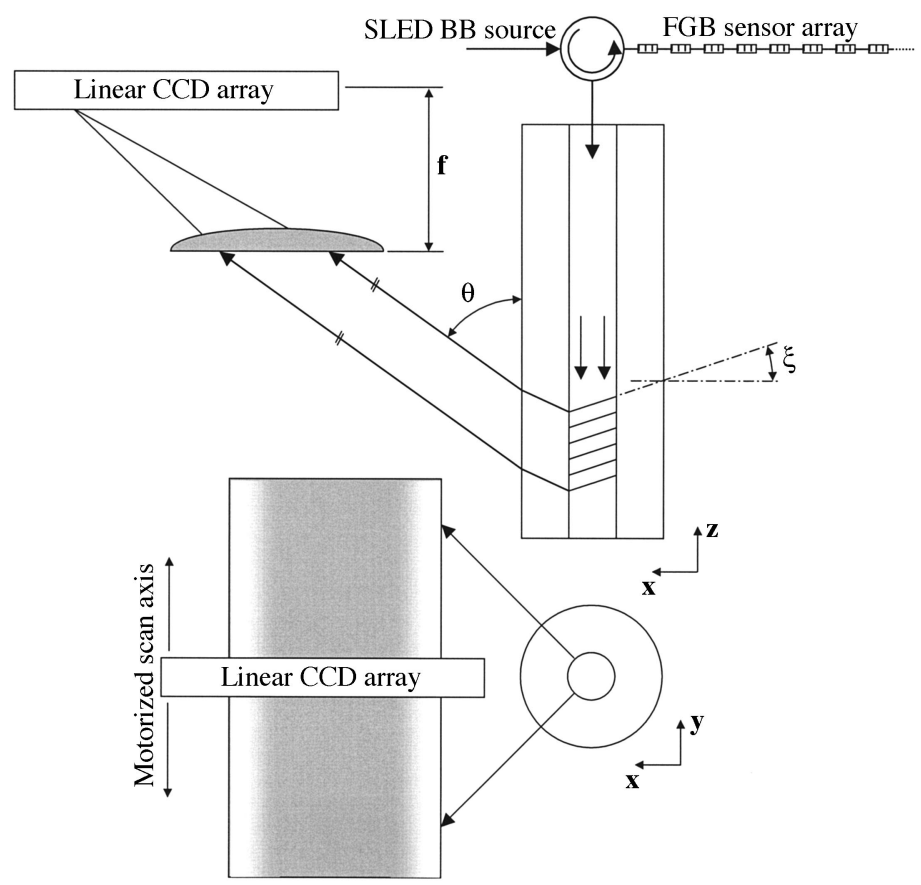

Fig. 3. Schematic diagram of the experimental apparatus.

lution may be utilized to implement a spectrometer function or to form the basis for low-cost WDM devices.

\section{Experimental Setup}

A BFBG was inscribed by the phase-mask method with a 244-nm Sabre FreD laser. A phase-mask angle of $7^{\circ}$ was used to write the grating in hydrogenated B-Ge codoped fiber, and the grating was subsequently annealed at $80^{\circ} \mathrm{C}$ for $48 \mathrm{~h}$ to stabilize the grating. The BFBG was clamped in a rotational stage to allow the direction of the radiated light to be controlled, and it was immersed in index-matching gel to remove cladding-air Fabry-Perot resonances from the grating spectrum. A coated Sony ILX511 CCD array consisting of $2048,14-\mu \mathrm{m}$ pixels was mounted on a motorized stage in the plane of the outcoupled light, as shown in Fig. 3. To make CCD devices sensitive to IR light it is necessary to apply a fluorescent coating; the resulting responsivity is invariably highly nonlinear. Figure 4 shows the responsivity for a Sony ILX511 array coated with Y2O2S:Er, Yb.

We easily achieved initial alignment by connecting the BFBG to a $5-\mathrm{mW}$ red $\mathrm{He}-\mathrm{Ne}$ source. After course alignment with the visible light radiated by the grating, the system was illuminated with a tunable laser with $-4.5-\mathrm{dBm}$ optical output. The CCD array was then scanned across the radiation profile of a 1511.2-nm source (chosen to coincide with the peak responsivity of Fig. 4). Figure 5 shows the beam profile recorded both with and without the cylindrical lens, positioned to focus in the $y$ plane. Figure 5(a) shows a contour plot that indicates the benefit of the cylindrical lens to the width of the beam profile, whereas Fig. 5(b) shows the three-dimensional (3D) profile and is a good indicator of the stark increase in optical power achieved when the lens is in place. The array was then positioned to bisect the point of highest optical power, the tunable laser was then scanned, and the position of each peak was noted.

Figure 6(a) shows how the spatial position of the beam profile depends on the wavelength of the radiation. It shows 11 beam profiles in $2-\mathrm{nm}$ steps for $\lambda=1500$ to $1520 \mathrm{~nm}$.

\section{A. System Calibration and Pixilation}

The system was calibrated by illumination of the BFBG with light from a narrow linewidth tunable laser. Each discrete wavelength illuminates an approximately Gaussian distribution of pixels on the

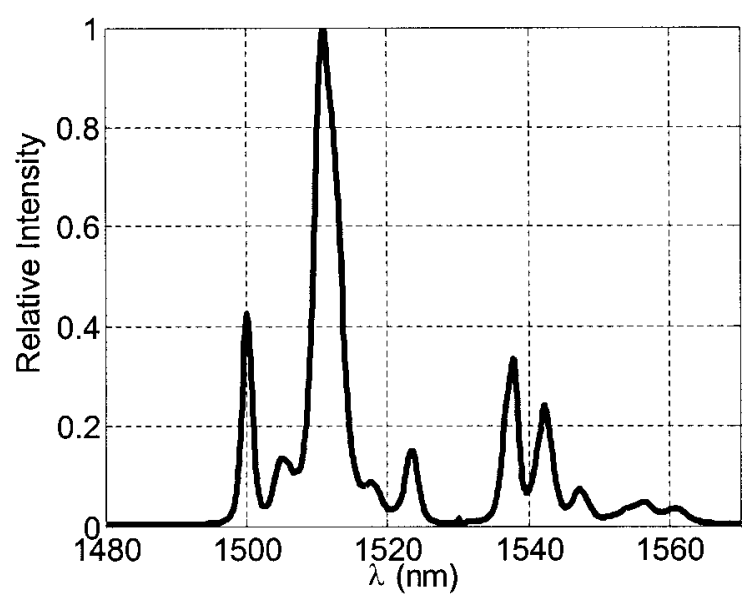

Fig. 4. Responsivity of a CCD array coated with $\mathrm{Y}_{2} \mathrm{O}_{2} \mathrm{~S}$ :Er, $\mathrm{Yb}$ to IR radiation. 


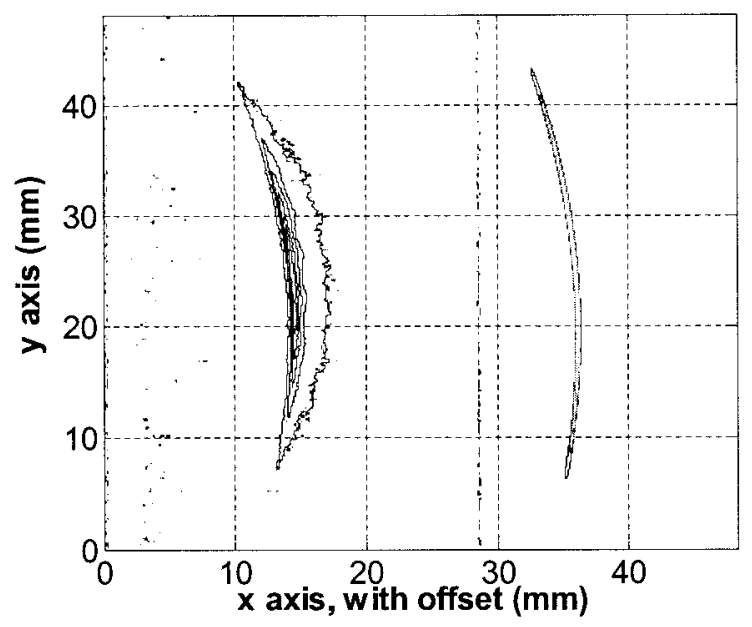

(a)

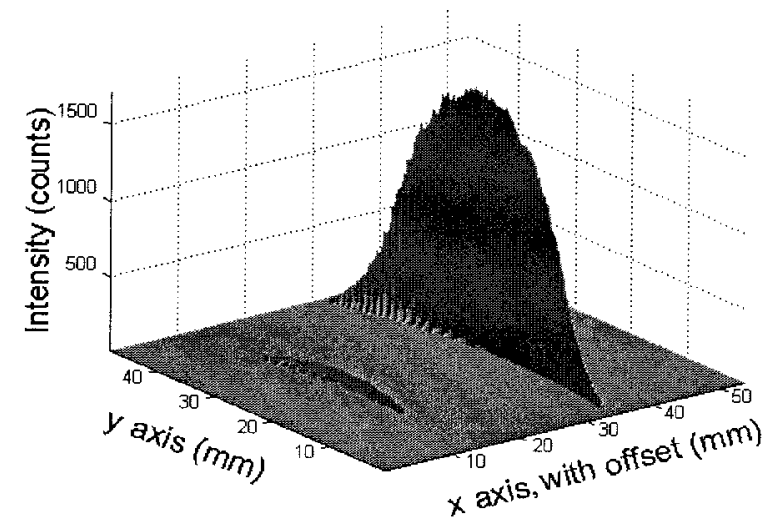

(b)

Fig. 5. (a) Contour plot showing the beam profile of outcoupled $1511.2 \mathrm{~nm}$ light from a BFBG with blaze angle $=8^{\circ}$. Shown without focusing (left) and with cylindrical lens in y-plane (right). (b) Surface plot showing the amplitude distribution of outcoupled $1511.2 \mathrm{~nm}$ radiation from a $\mathrm{BFBG}$ with blaze angle $=8^{\circ}$. Shown without focusing (left) and with cylindrical lens in y-plane (right).

CCD array. By plotting the most intensely illuminated pixel as a function of wavelength one may quickly build up an accurate transfer function relating wavelength to position, as shown by Fig. 6(b). It shows that the relation is linear, to a good approximation. Naturally, the gradient of this transfer function depends on the distance from the BFBG, and hence the focal length of the cylindrical lens shown in Fig. 3(f). Figure 6(a) shows the transfer function for two lenses with $f=50 \mathrm{~mm}$ and $f=150 \mathrm{~mm}$ for $\Delta \lambda$ steps of $10 \rho \mathrm{m}$.

Pixilation resulting from the small change in wavelength being insufficient to illuminate the next adjacent pixel can clearly be seen in the inset of Fig. 6(b). The effect of finite pixel width may be greatly reduced by use of the simplistic centroid detection algorithm ${ }^{17}$ (CDA) wherein an amplitude weighted average of the pixels' intensity is taken, i.e.,

$$
\hat{i}=\frac{\sum_{1.2048} x_{i} y_{i}}{\sum_{1.2048} y} \quad \text { for all } y_{i}>\text { threshold }
$$

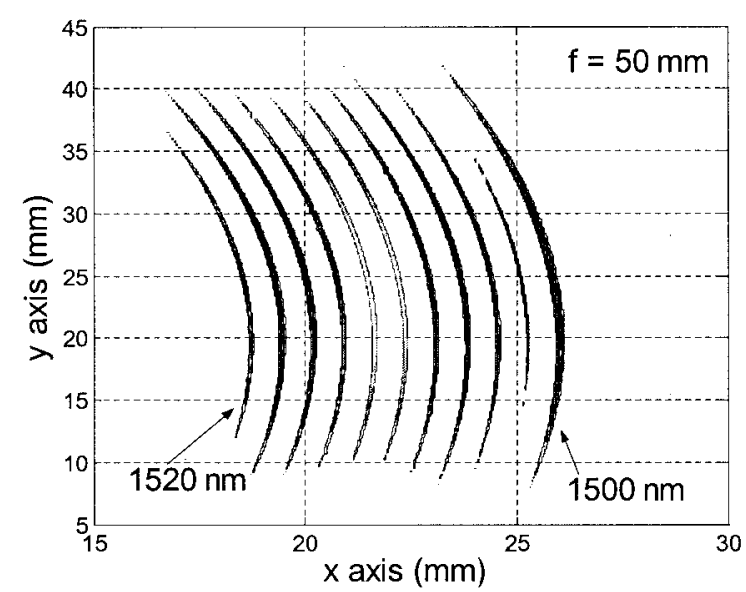

(a)

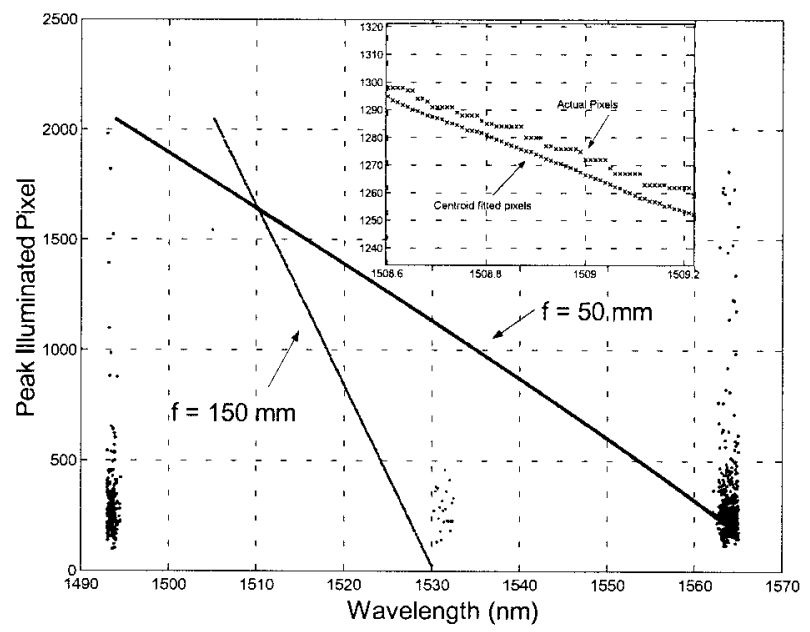

(b)

Fig. 6. (a) Contour plot showing the spectral dependence of the radiation profile for 1500 to $1520 \mathrm{~nm}$ in 2-nm steps. (b) Diagrammatic representation of the transfer function describing the position of radiation modes for discrete wavelengths. Inset: Pixelation correction resulting from the CDA.

where $x_{i}$ represents is the pixel number, $y_{i}$ is the intensity of each pixel and $\hat{i}$ is the CDA fitted $\lambda_{\mathrm{BR}}$ value. The CDA may be performed in real time as the data is captured. The inset of Fig. 6(b) clearly shows the significant reduction in pixilation.

\section{B. System Bandwidth and Resolution}

The ultimate system bandwidth is limited by the length of the CCD array and the bandwidth of the BFBG's radiation modes. For a CCD array of a given length and a BFBG of given spectra, the bandwidth of the system is a function of the distance from the grating to the array. The physical resolution may then simply be calculated as the bandwidth divided by the number of pixels. Note that datainterpolation techniques (e.g., CDA) reduce the physical resolution significantly. For the two lenses used in Fig. 6(a), the system parameters may be summarized as per Table 1. 
Table 1. System Parameter Summary for Two Lenses of Different Focal Length

\begin{tabular}{cccc}
\hline $\begin{array}{c}\text { Focal } \\
\text { Length } \\
(\mathrm{mm})\end{array}$ & $\begin{array}{c}\text { Dynamic } \\
\text { Range } \\
(\mathrm{nm})\end{array}$ & $\begin{array}{c}\text { Gradient } \\
(\mathrm{nm} / \mathrm{mm})\end{array}$ & $\begin{array}{c}\text { Physical } \\
\text { Resolution } \\
(\rho \mathrm{\rho m} / \mathrm{pixel})\end{array}$ \\
\hline 50 & 70 & 2.71 & 38 \\
150 & 25 & 0.89 & 12 \\
\hline
\end{tabular}

\section{Experimental Results}

A WDM sensor array of nominally four elements was constructed in standard fiber with $\lambda_{\mathrm{BR}}$ values chosen to coincide with the peak responsivities of Fig. 4. Initial experiments required the use of a scanning tunable laser as the light source because the light levels incident to the CCD array when illuminated from a broadband source were insufficient to stimulate the device. Later experiments incorporated an extra two cylindrical lenses, placed to focus in the $x$ plane, which consolidated the radiation profile into a narrow point of sufficient intensity to permit a broadband superluminescent light-emitting diode (SLED) to be used as the light source. The spectrum of this source is shown in Fig. 7.

Strain and Temperature Sensing with Broadband Source: The grating array was connected to the output of an optical circulator, as shown in Fig. 3, and illuminated by a SLED light source running at maximum power (refer to Fig. 7). The return port of the circulator (port 3) was then connected to the BFBG. The light was radiated onto the CCD array and the waveform captured by personal computer (PC). The transfer function relating illuminated pixel to wavelength was then applied in the software to derive the spectrum of the light reflected from the grating array.

In separate experiments, gratings from the array were monitored in strain and in temperature changes. For each discrete change of measurand, 10 readings of $\lambda_{\mathrm{BR}}$ were calculated with the CDA, and their averages were plotted. Figure 8(a) shows the results for an increase in strain of up to $1400 \mu \varepsilon$,

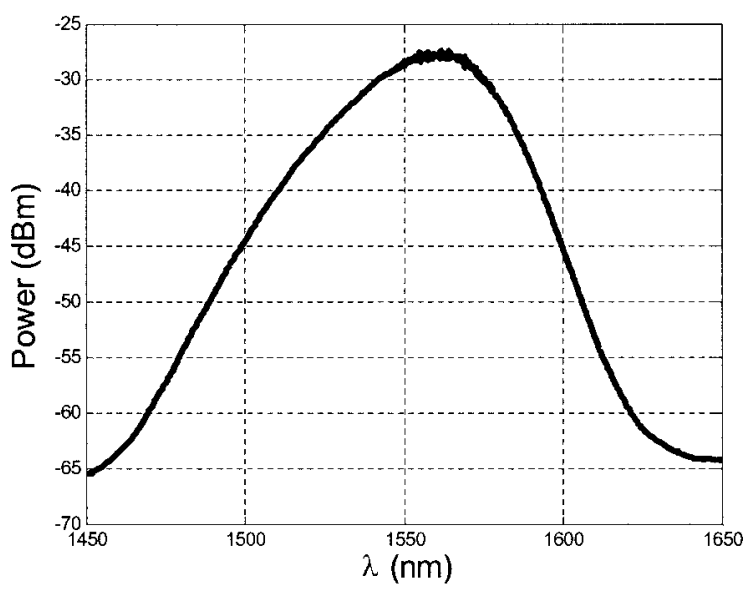

Fig. 7. Spectrum of the SLED light source used to illuminate the system.

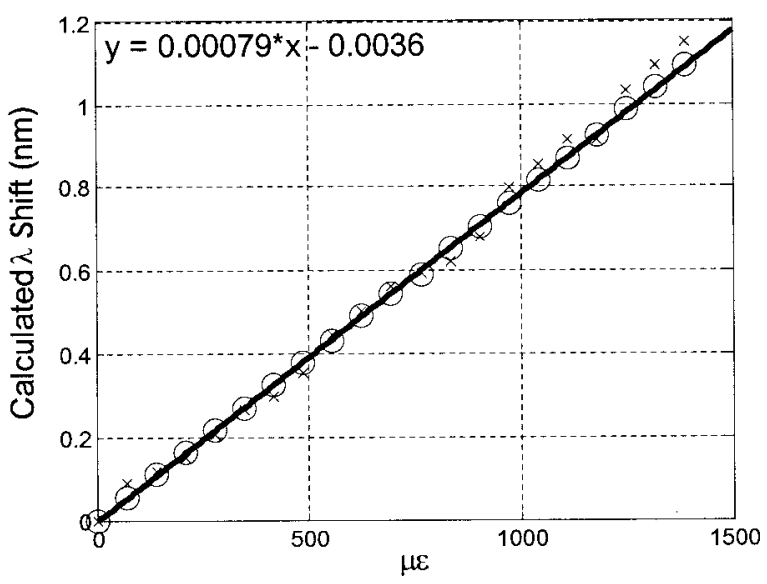

(a)

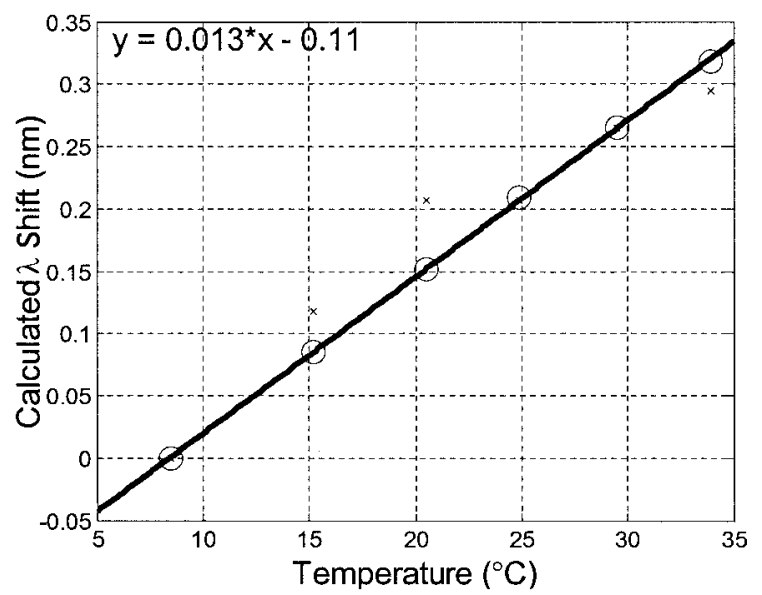

(b)

Fig. 8. Detected (a) strain- and (b) temperature-induced $\lambda_{\mathrm{BR}}$ shifts with the BFBG-CCD apparatus.

whereas Fig. 8(b) shows the results for a temperature shift of $30^{\circ} \mathrm{C}$. Figures $8(\mathrm{a})$ and $8(\mathrm{~b})$ show an rms deviation from a linear trend of 3.7 and $1.7 \mathrm{\rho m}$, respectively.

The points marked with ' $O$ ' in Fig. 8 represent the values of $\lambda_{\mathrm{BR}}$ calculated with the CDA, whereas those marked with ' $x$ ' are the value calculated simply by looking for the point of maximum optical reflection. The difference in accuracy is clearly demonstrated by these two datasets.

\section{System Performance Analysis}

\section{A. Signal-to-Noise Ratio}

Figure 9(a) shows the spectrum of one of the sensor fiber Bragg gratings (FBGs) used in the previous section. The SNR was calculated from the relative intensities of the FBG peak and the maximum noise level of the system (after correction in the software for dark current). To show the effect of integration time on SNR, the system was set up to monitor the reflection from a sensor FBG, held at constant temperature with constant illumination level, whereas the integration time was varied. Figure 9(b) shows 


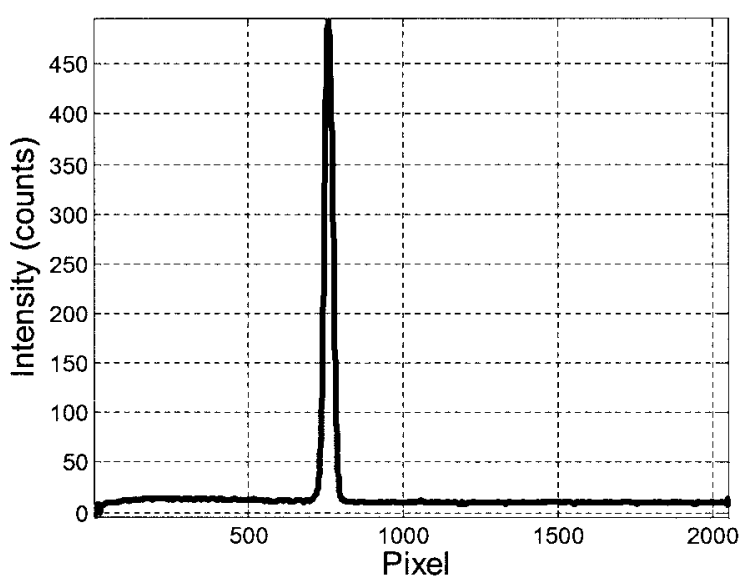

(a)

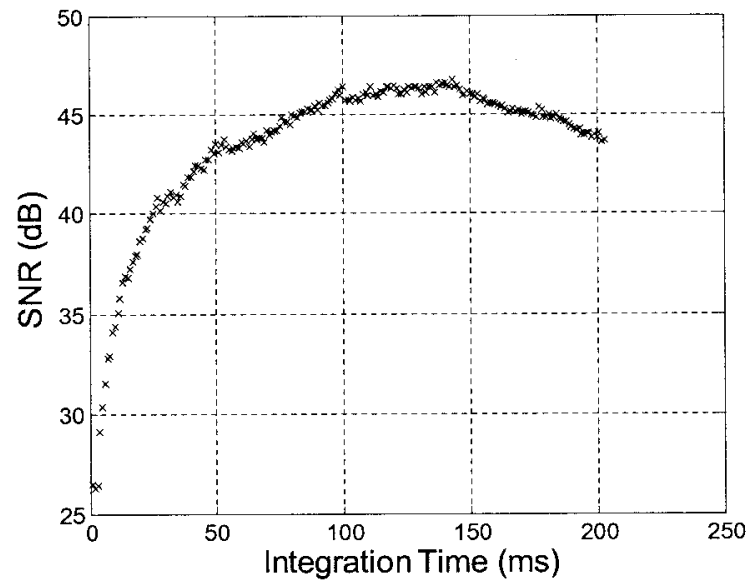

(b)

Fig. 9. (a) CCD output spectrum when illuminated with a sensor FBG and SLED broadband source. (b) SNR for increasing integration time.

the SNR in decibels as a function of integration time. At approximately $t=120 \mathrm{~ms}$, the SNR begins to decrease as a result of the signal saturating the array.

\section{B. Drift}

Drift is another important factor to consider when designing a sensor interrogation system. Values that should remain constant over time, but show change, are a contributing factor to overall system error. To model this, we used the system to monitor the reflected spectrum from a sensor FBG, held at constant temperature, for approximately $45 \mathrm{~min}$ with a sample rate of $1 \mathrm{~Hz}$.

Figure 10(a) shows the recorded value of $\lambda_{\mathrm{BR}}$ against time. The large variations are believed to be caused by temperature changes in either the environment surrounding the BFBG or the TEC controlled sensor. To ascertain the fluctuations in the reading made by the system, we mathematically filtered out these large variations and have shown them in Fig. 10(b). Figure 10(c) shows a histogram displaying the distribution of these filtered readings that form a clear normal distribution. The rms deviation from

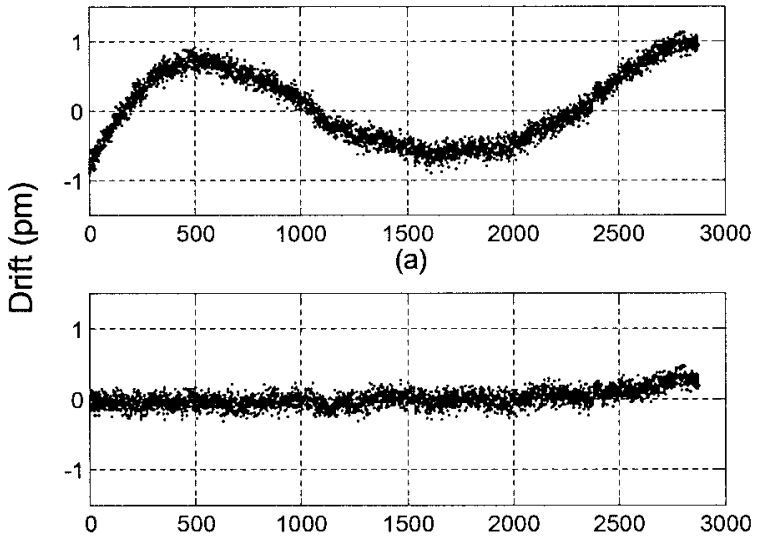

(b)

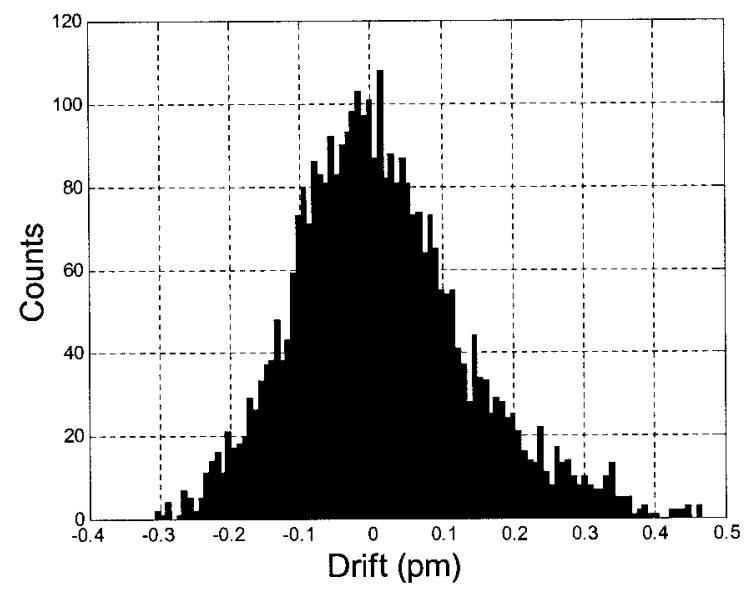

(c)

Fig. 10. Drift over time of the recorded $\lambda_{\mathrm{BR}}$ value of a sensor FBG measured with the BFBG-CCD system: (a) raw data and (b) Filtered data for external temperature variations. (c) Histogram to show the distribution of the recorded values of $\lambda_{\mathrm{BR}}$ over a 45-min period.

the mean of this histogram is a mere $0.12 \rho \mathrm{m}$, which indicates that the system is inherently stable.

\section{Polarization Independence}

Polarization sensitivity affects the performance of a sensor interrogation system because random changes in polarization state may be interpreted as a measurand change. To ascertain the sensitivity of the system to these changes in polarization state, we used a tunable laser to simulate the peak formed by the reflection of light from an FBG sensor on the CCD array. This radiation was passed through a PCcontrolled polarization state adjuster before reaching the BFBG-CCD system. The polarization-state adjuster was set to scan all possible permutations over a 12-s period. The peak position and amplitude was then logged every $250 \mathrm{~ms}$ for a 20 -min period. Figure 11 clearly shows that although the intensity of the radiation varies according to polarization state, its spatial position remains constant, thus indicating polarization insensitivity for sensor gratings illuminated with a broadband source since only a snapshot is required. 


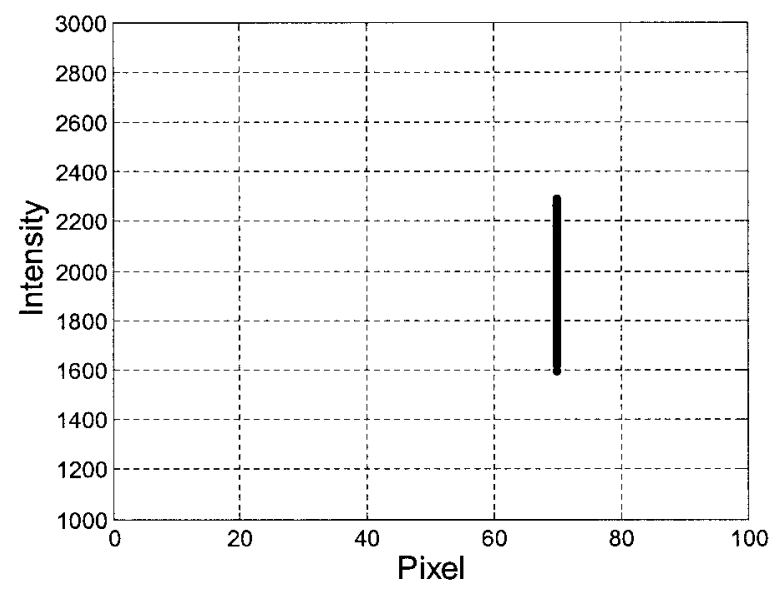

Fig. 11. Peak position of a sensor for all possible polarization states; demonstrating the polarization independence of the BFBGCCD detection system.

Theoretical results may be used to simply explain this effect. 18 Figure 12 shows the polar plot of the simulated far-field image of radiation modes from a $5^{\circ}$ grating for 633 and $1530 \mathrm{~nm}$ light at $0^{\circ}$ and $90^{\circ}$ linear polarizations. It is clear that at shorter wavelengths, the polarization state of the incident radiation has a significant effect on the far image, but as the wavelength approaches the Bragg wavelength of the grating the effect is negligible and only significantly alters the intensity distribution within the main lobe.

\section{Wavelength Division Multiplexed Channel Separation}

For a multiplexed system, the minimum possible spacing of sensors is an important factor because it

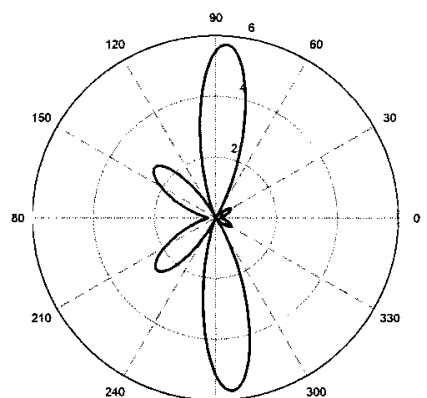

(a)

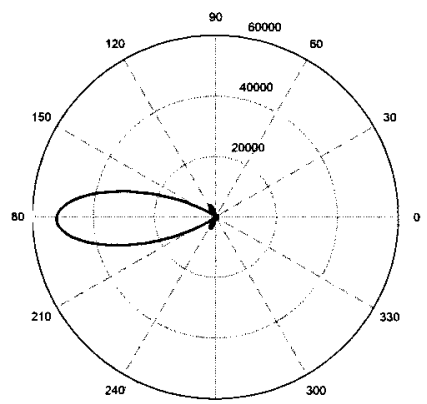

(c)

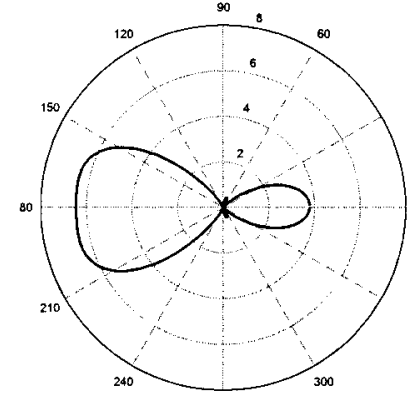

(b)

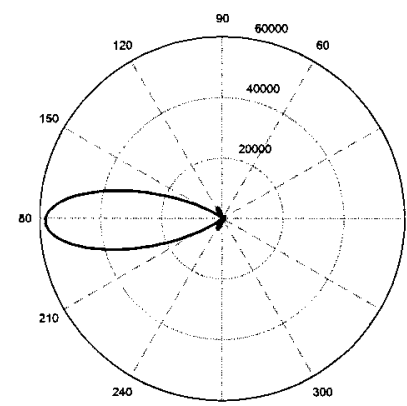

(d)
Fig. 12. Simulated far-field images for $0^{\circ}$ and $90^{\circ}$ polarized at 633- [(a) and (b)] and 1560-nm [(c) and (d)] radiation.

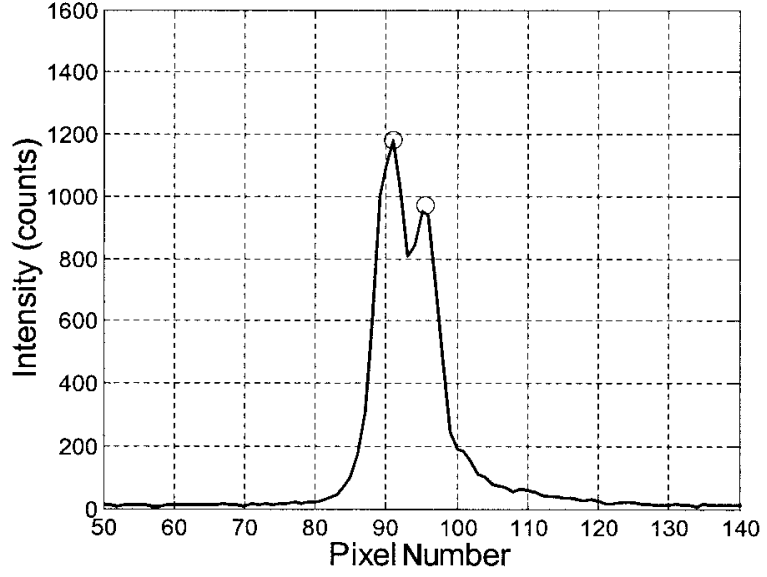

(a)

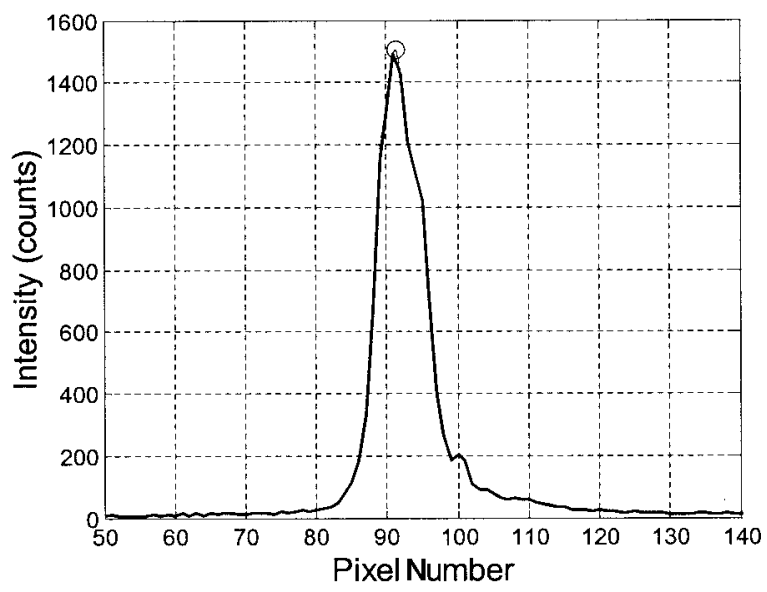

(b)

Fig. 13. Two received sensors separated (a) by 100 pm (WDM separation of $100 \rho \mathrm{m}$ ) and (b) by $50 \mathrm{\rho m}$ (WDM separation of $50 \rho \mathrm{m}$ ).

determines the number of sensors per channel. Clearly, as signals begin to overlap, trade-offs must be made between SNR and WDM spacing. To demonstrate the ultimate limit of this system in terms of WDM separation, we used two tunable laser sources to simulate sensor FBGs with $0.5-\mathrm{nm}$ linewidth. One was held at a constant value, whereas the other was scanned to cross its spectral location. The point at which the two peaks became indistinguishable was defined as the minimum WDM spacing. Figure 10 shows two frames from this experiment; Fig. 13(a) shows the point when the two sources are separated by $100 \rho \mathrm{m}$, and Fig. 13(b) shows them at 50-pm separation.

Note that minimum WDM separation depends largely on the linewidth of the FBG and the separation of the CCD detector from the BFBG. For this instance with $\Delta \lambda$ at $0.5 \mathrm{~nm}$ and $f=50 \mathrm{~mm}$, the minimum WDM channel separation has been defined as $75 \rho \mathrm{m}$.

\section{Conclusions}

We have performed the first comprehensive practical investigation into the application of BFBGs as dis- 
persive spectral interrogators for WDM in-fiber optical sensors. Our results show that a BFBG may be used in conjunction with a linear CCD array to produce an extremely low-cost sensor interrogation system. We have shown that light from a broadband source, reflected by a sensor grating is sufficiently intense to be detected at the side of a BFBG. We have also shown that the exit angle of these radiation modes is highly $\lambda$ dependent. We present results that show the system may be used to interrogate a sensor array with WDM spacing at $75 \mathrm{\rho m}$, SNR greater than $45 \mathrm{~dB}$, and bandwidth of $70 \mathrm{~nm}$. We further show polarization insensitivity and drift with time of only $0.1 \rho \mathrm{m}$.

This research was carried out under the United Kingdom Department of Trade and IndustryEngineering and Physical Science Research Council LINK project EMPIRE, and we acknowledge our project partners BAE Systems, Indigo Photonics Ltd, and Deutsch Ltd for their technical support and useful discussions.

Acknowledgment is also owed to the Photonics Application Centre (PAC), Photonics Cluster (United Kingdom) for the provision of some equipment.

A. G. Simpson further acknowledges the generous studentship of the UK EPSRC and BAE Systems.

\section{References}

1. G. Meltz, W. Morey, and W. Glenn, "In-fibre Bragg grating tap," in Optical Fiber Communication Conference (Optical Society of America, Washington, D.C., 1990), TuG1.

2. P. S. Westbrook, K. S. Feder, P. I. Reyes, P. Steinvurzel, B. J. Eggleton, R. G. Ernst, L. A. Reith, and D. M. Gill, "Application of fiber Bragg grating filter/tap module to a wavelength-locked low-chirp directly-modulated $10 \mathrm{~Gb} / \mathrm{s}$ RZ transmitter," in $O p$ tical Fiber Communication Conference and Exhibit, 2002 (IEEE, Piscataway, N.J., 2002), pp. 680-682.

3. R. Kashyap, R. Wyatt, and R. J. Campbell, "Wideband gain flattened erbium fibre amplifier using a photosensitive fibre blazed grating," Electron. Lett. 29, 154-156 (1993).

4. H. Labidi, C. Debarros, R. Letteron, and Riant, "Slanted Bragg grating with ultra-low polarization dependent loss," in Optical
Fibre and Communication Conference and Exhibit, 2002 (IEEE, Piscataway, N.J., 2002), p. 113.

5. Y. Liu, L. Zhang, and I. Bennion, "Fabricating fibre edge filters with arbitrary spectral response based on tilted chirped grating structures," Meas. Sci. Tech. 10, L1-L3 (1999).

6. J. L. Wagner, T. A. Strasser, J. R. Pedrazzini, J. DeMarco, and D. J. DiGiovanni, "Fibre grating optical spectrum analyzer tap," in European Conference on Optical Communication (ECOC'97) (IEE, Edinburgh, 1997), pp. 65-68.

7. T. Erdogan, "Fibre grating spectra," J. Lightwave Tech. 15, 1277-1294 (1997).

8. T. Erdogan and J. E. Sipe, "Tilted fiber phase gratings," J. Opt. Soc. Am. A 13, 296-313 (1996).

9. K. S. Lee and T. Erdogan, "Fiber-mode conversion with tilted gratings in an optical fiber," J. Opt. Soc. Am. A 18, 1176-1185 (2001).

10. K. S. Lee and T. Erdogan, "Fiber-mode coupling in transmissive and reflective tilted fiber gratings," Appl. Opt. 39, 13941404 (2000).

11. K. S. Lee and T. Erdogan, "Transmissive tilted gratings for LP0.1-to-LP11 mode coupling," IEEE Photon. Technol. Lett. 11, 1286-1288 (1999).

12. Y. Koyamada, "Analysis of core-mode to radiation-mode coupling in fiber Bragg gratings with finite cladding radius," J. Lightwave Technol. 18, 1220-1225 (2000).

13. K. Zhou, A. G. Simpson, L. Zhang, and I. Bennion, "Sidedetection of strong radiation mode out-coupling from blazed FBGs in single- and multi-mode fibers," Photonics Technol. Lett. 15, 936-938 (2003).

14. K. Zhou, A. G. Simpson, L. Zhang, and I. Bennion, "Twodimension optical power distribution of side-out-coupled radiation from tilted FBGs in multi-mode fibre," Electron. Lett. 39, 651-653 (2003).

15. A. G. Simpson, K. Zhou, L. Zhang, and I. Bennion, "High accuracy interrogation of a WDM FBG sensor array using radiation modes from a B-FBG," in Bragg Gratings, Photosensitivity and Poling in Glass Waveguides (Optical Society of America, Washington, D.C., 2003), pp. 82-84.

16. S. J. Mihailov, R. B. Walker, P. Lu, H. Ding, X. Dai, C. Smelser, and L. Chen, "UV-induced polarisation-dependent loss (PDL) in tilted fibre Bragg gratings: application of a PDL equaliser," IEE Proc.-Optoelectron 149, 211 (2002).

17. A. Ezbiri, S. E. Kanellopoulos, and V. A. Handerek, "High resolution instrumentation system for fibre-Bragg grating aerospace sensors," Opt. Commun. 150, 43-48 (1998). 